PRELIMINARY MEASUREMENTS OF PLASMA FLUCTUATIONS

IN AN 8-CM MERCURY ION THRUSTER

by John S. Serafini and Shigeo Nakanishi

Lewis Research Center

Cleveland, Ohio 44135

TECHNICAL PAPER to be presented at

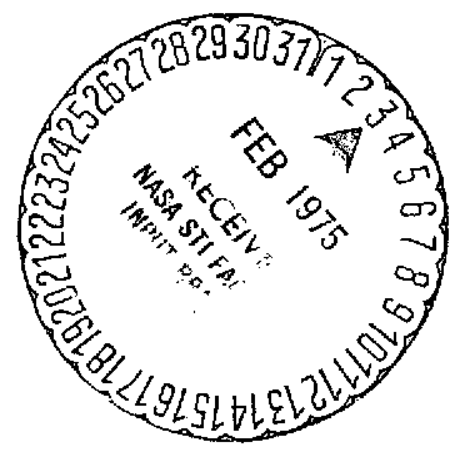

Eleventh Electric Propulsion Conference sponsored by American Institute of Aeronautics and Astronautics New Orleans, Louisiana, March 19-21, 1975 


\title{
PRELIMINARY MEASUREMENTS OF PLASMA FLUCTUATIONS IN AN 8-CM MERCURY ION THRUSTER
}

\author{
John S, Serafini and Shigeo Nakanishi \\ National Aeronautics and Space Administration \\ Lewis Research Center \\ Cleveland, Ohio
}

\begin{abstract}
The rms magnitude, spectra, and crosscorrelations for the fluctuations in the beam current, the neutralizer keeper current, and the discharge current and voltage were measured for an 8-cm diameter dished grid ion thruster for a beam current of 72 milliamps. The effects on the fluctuations of varying several neutralizer parameters, including the keeper hole diameter, the keeper current, and the flow rate were studied. Varying the neutralizer keeper hole diameter resulted in a variation in the ratio of the rms magnitude of the fluctuation to the time-mean of the beam current from 0.02 to 0.67 . The ratio of the rms magnitude of the fluctuations to the time-mean neutralizer keeper current was found to depend significantly on the neutralizer timemean keeper current, the flow rate, and keeper hole diamcter. The maxima of the spectra of the beam current fluctuations did not depend on the discharge fluctuations. From the spectral and crossm correlation results, the discharge current fluctuations did not directly contribute to the beam current fluctuations. The rms magnitudes, spectral and cross-correlation results of the neutralizer keeper current fluctuations indicate that the neutralizer contributions to the beam fluctuations could be small (for good neutralizer-to-beam coupling) but not negligible and appear mostly in the higher frequency range measured.
\end{abstract}

\section{Introduction}

The 8-cm diameter mercury electron bombardment ion thruster is designed to provide stationkeeping and attitude control of geosynchronous spacecraft. (1) All

flight tests to date have not indicated any electromagnetic interference caused by thruster fluctuations and noise. However, because of the importance of this area, the further study of thruster fluctuations and noise is warranted. A mercury ion thruster can produce fluctuations about the mean ion beam current, in the discharge plas$\mathrm{ma}$, and possibly within the power conditioner itself. ${ }^{(2)}$ These fluctuations can occur over a broad frequency spectrum.

This paper gives the initial and first-known results of measurements of fluctuations on an 8-cm mercury ion thruster. The investigation included observing the effects on the fluctuations of varying several neutralizer parameters, particularly the neutralizer keeper hole size, the keeper current, and flow rate. The $8-\mathrm{cm}$ mercury ion thruster utilizes a dished grid ion extraction system. It was designed to operate at 3000 seconds specific impulse at thrust levels of 4.4 to $8.9 \mathrm{mN}$ (1) to to $2 \mathrm{mlb}$ ).

New measurements wcre obtained of the fluctuations of the ion beam current, the neutralizer keeper current, and the voltage and current of the discharge. Several aspects of these fluctuations were recorded, (2) They included (1) the overall magnitude or intensity given as the rms (root-mean-square) magnitude, (2) the frequency spectral distribution, and (3) cross-correlations of the fluctuations.

\section{Apparatus and Experimental Techniques}

\section{Thruster}

A photograph of the $8-\mathrm{cm}$ diameter mercury ion thruster mounted on the vacuum port cover flange is shown in Fig. 1. The thruster is designed to operate at a specific impulse near 3000 seconds and thrust levels of 4.4 to $8.8 \mathrm{mN}$ ( 1 to $2 \mathrm{mlb}$ ). The thruster design ${ }^{(1)}$ incorporates high perveance ion extraction grids, permanent magnets, and hollow cathodes with rolled tantalum foil inserts for both the discharge chamber and neutralizer. The ncutralizer keeper is of the enclosed type whose hole size was incrementally increased from 0.081 to $0.257 \mathrm{~cm}$ in this investigation.

\section{Thruster Power Supply}

A block diagram and details of the laboratory test power console for the thruster are given in Ref. 1 . Only those specifications believed to be pertinent to the thruster current and voltage fluctuation measurements will be summarized here. A line regulator was provided to eliminate line transients from the primary power buss.

The net accelerating potential was provided by a voltage and current regulated supply with automatic crossover. Regulation was better than $0.01 \%$ of full scale. The output impedance was approximately $4 \mu \mathrm{F}$.

The discharge chamber was driven by a 100-volt de, 0- to 1.5-amp supply with a 0- to 1.5-amp current regulator in its output circuit. The output circuit of the current regulator had only stray capacitance associated with it and therefore was capable of delivering constant. 
current to the discharge well into the kilohertz frequency range.

The neutralizer keeper supply was a dc current regulated supply adjustable from 0 to $0.5 \mathrm{amp}$ at a nominal 34 volts. The neutralizer supply common was isolated from facility ground, and a clamp circuit consisting of back-to-back gas discharge tubes was a safety provision to maintain the neutralizer floating potential to within 150 volts of ground potential. Measured impedances between the neutralizer common and facility ground were 1. $6 \mu \mu \mathrm{F}$ without the power console and $0.52 \mu \mathrm{F}$ in parallel with about $100 \mathrm{~K}$ ohms with the power console connected to the thruster.

\section{Thruster Operation}

The thruster was operated in a 1.5-m diameter by 4- $m$ long vacuum chamber at an environmental pressure of about $3 \times 10^{-6}$ Torr. Horizontally translatable tracks were used to project the thruster past a $30,5-\mathrm{cm}$ gate valve opening and into the chamber, thus minimizing possible wall effects upon the ion beam and beam neutralization.

The nominal operating conditions of the thruster for a beam current of 72 milliamps at 1250 volts net accelerating potential and $\mathbf{- 5 0 0}$ volts accelerator potential were: discharge voltage, 38 volts; discharge current, $0.70 \mathrm{amp}$; keeper discharge, $0.26 \mathrm{amp}$ at 10 volts. The cathode flow rate was held at about 90 milliamps $\mathrm{Hg}^{+}$ equivalent.

The neutralizer keeper discharge was set for 0.45 amp (except when varied as a test variable) at 13 to 20 volts depending upon the keeper hole size and flow rate. The neutralizer flow rate was varied over a range from 1.75 to 10,0 equivalent milliamps.

\section{Transducers}

Two current probes $(0,64-\mathrm{cm}$ diameter molybdenum planar) were used to sense ion beam current fluctuations (see Figs. 1 and 2). One probe was mounted on the flange supporting the thruster and it could be rotated about its support arm to allow measurements to be made at up to $30 \mathrm{~cm}$ radially from the centerline of the thruster. The other probe was mounted on the tank wall and was moved vertically through the beam at an axial distance of $12,7 \mathrm{~cm}$ from the accelerator grid of the thruster. Both probes were moved through the centerline of the thruster. Both probes were biased at -20 volts by means of shielded variable-voltage bias supplies to permit operation of the probes in the ion-collection mode.

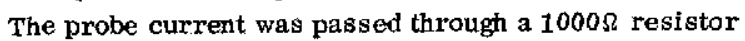
connected to ground. The voltage drop across the resistor was then used to measure current. The time-mean voltages were monitored on de digital voltmeters and the time-varying voltages were fed into cathode follow- ers having a high input impedance. An ac coupled amplifier provided a suitable output of the thme-varying signal. It was possible to inspect portions of the frequeney content of the broadband data by the use of highpass band or low-pass band frequency filtering.

Current loop probes were used to sense emission current, total ion beam current, and neutralizer keeper current fluctuations. These probes were placed around the appropriate current-carrying conductors from the power supply to the thruster. The discharge voltage fluctuations were sensed by current loop about a lead having a $1003 \Omega$ noninductive resistor across the discharge supply leads before being connected to the thruster. The sensitivity of the current loop was 2 milliamps per millivolt, respectively.

The overall gain and frequency response of the transducers used was checked and measured. For the same input signal the phase difference (of importance in the correlation studies as described in Ref. 2) between the output signals for the two probe systems was negligibly small over the frequency range of interest. For further details of the calibration of the data system refer to Ref. 2.

\section{Analysis of Time-Varying Data}

The magnitudes of the timo-varying output signals from the ion beam probe and current-loop probe systems were measured on meters which gave the true root-mean-square magnitude. The amplitude as a function of frequency was measured by the use of three spectrum analyzers over ranges such as 0 to $3.5 \mathrm{MHz}$, 0 to $2 \mathrm{MHz}, 0$ to $1 \mathrm{MHz}, 0$ to $100 \mathrm{kHz}$, and 0 to $10 \mathrm{kHz}$. The signals from the spectrum analyzers were fed to $x-y$ recorders. The final type of processing of the time-varying output signale, was the measurement of the space-time or cross-correlations as well as the auto-correlations of fluctuations in the beam. A brief description of this technique and its implications are given in appendix B of Ref. 2. Also, measurements of the cross and auto-correlations between fluctuations sensed by the current loop probes were obtained.

\section{Results and Discussion}

This section will present the results on rms magnitude, spectral data and the correlations in that order.

\section{rms Magnitudes}

Figure 3 shows a radial variation of the time-mean $\overline{I(t)}$ and root-mean square magnitude $\sqrt{i^{2}(t)}$ of the ion beam probe current fluctuations sensed at a fixed axial distance from the grid. (All symbols are also defined in the appendix.) In this and all other figures, the functional dependence on time, $t$, will be omitted in the 
notation and thus $\overline{I(t)} \rightarrow \bar{I}$ and $\sqrt{\overline{i^{2}(t)}} \rightarrow \sqrt{i^{2}}$. The data in Fig. 3 are given for the standard operating condition of this thruster (and for all of the results presented herein). These are an ion beam current, $\mathrm{J}_{\mathrm{B}}$, of 72 milliamps, a discharge voltage, $\Delta \mathrm{v}_{\mathrm{I}}$, of 38.0 volts and a discharge current, $J_{E}$, of 0.70 amp. The axial displacement of the probe from the accelerator grid was $12.7 \mathrm{~cm}$ for all of the results presented herein. From the curves in Fig. 3 it is seen that the trend of the rms values of the fluctuations and the time-mean current are generally similar. The ratio of the rms to the time-mean current at the peak of the time-mean curve is 0.026 and at a radial distance, $r$, of $4 \mathrm{~cm}$ (from the poak it is 0.030 ).

The manner in which the neutralizer provides electrons for the beam and thus is "coupled" to the beam has been of concern in previous 8-cm mercury ion thruster studies (i.e., Ref. 1). Consequently, it was considered to be an important part of the present investigation to look at how the fluctuations in the neutralizer affected both the tima-varying and the time-mean aspects of the coupling of the neutralizer with the beam. In Fig. 4 the effect of the neutralizer fluctuations on the beam fluctuations is considered. Figure 4 shows the rms magnitude of the neutralizer keeper current fluctuations as a function of the ratio of the rms magnitude to the time-mean beam currents for the range of neutralizer keeper hole diameters investigated from 0.081 to $0.257 \mathrm{~cm}$. The data in Fig, 4 includes values of $\mathrm{J}_{\mathrm{NK}}$ from 0.2 to 0.45 amp and neutrailzer cathode flow rates from 1.75 to 10 milliamps.

The desirable conditions of operation obviously should include those where the ratio of the rms magnitude to the time-mean beam current are a minimum. Thus, the points which extend from values of $\sqrt{j_{B}^{2}} \mid \bar{J}_{B}$ 0.10 to 0.67 , are not desirable. As these values get larger, the beam becomes more unstable, which results in the production of "beam noise." As is seen in Fig. 4, there is a considerable set of points which have values of $\sqrt{\overline{j_{B}^{2}}} / \bar{J}_{\mathrm{B}}$ less than 0.10 and these points lie near the vertical portion of the curve. With one exception all these points are for neutralizer keeper hole diameters, $\mathrm{d}_{\mathrm{N}}$, of $0.157 \mathrm{~cm}$ or greater. Although the neutralizer keeper current fluctuations cover a range greater than a factor of 2 , the ratio $\sqrt{\overline{j_{B}^{2}}} \mid \bar{J}_{B}$, remains under 0.07 . For this set of values of $\mathrm{d}_{\mathrm{N}}$ equal to or greater than $0.157 \mathrm{~cm}$, the values of $\mathrm{J}_{\mathrm{NK}}$ and the neutralizer flow rate were $0.45 \mathrm{amp}$ and 7.0 milliamps, respectively.

To examine the time-mean neutralizer-beam coupling the rms magnitude of the neutralizer keeper current fluctuations is presented as a function of the neutralizer floating potential, $\mathrm{V}_{\mathrm{g}}$ in Fig. 5. A correspondence is noted between the neutralizer floating potential values in Fig. 5 and the $\sqrt{\mathrm{j}_{\mathrm{B}}^{2}} / \bar{J}_{\mathrm{B}}$ values in Fig. 4 Where the coupling is good $\left(\left|\mathrm{V}_{\mathrm{g}}\right|\right.$ equal to $12.0 \mathrm{~V}$ or less), the values of $\sqrt{\overline{j_{B}^{2}}} / \bar{J}_{B}$ are less than 0.07 . Where the coupling to the beam current is not as good $\left(\left|V_{g}\right|>12.0 \mathrm{~V}\right)$, the values of $\sqrt{\mathrm{j}_{\mathrm{B}}^{\overline{2}}} \mid \overline{\mathrm{J}}_{\mathrm{B}}$ increase correspondingly. In other words, where the time-mean or steady-state coupling of the neutralizer with beam current is good, a minimum fluctuation in the beam current is obtained or the time-varying coupling is also good,

In Fig. 6 is presented the ratio of the rms magnitudes of the fluctuations to the time-mean currents for both the neutralizer keeper current and the beam current as a function of the neutralizer flow rate, $\dot{\mathrm{m}}_{\mathrm{N}}$ in milliamps for $J_{\mathrm{NK}}$ of $0.45 \mathrm{amp}$ and $\mathrm{d}_{\mathrm{N}}$ of $0.193 \mathrm{~cm}$. In this particular case, the effect of varying $\dot{\mathrm{m}}_{\mathrm{N}}$ is comparable for both the beam and neutralizer keeper current fluctuations. At low neutralizer flow rates, however, the neutralizer fluctuations begin to increase more rapidly than the beam fluctuations. The particular shape of each curve was found to be dependent on the value of $\mathrm{J}_{\mathrm{NK}}$ and the neutralizer keeper hole diameter.

Figure 7 shows the ratio of the rms magnitudes to the time-mean currents for both the neutralizer keeper and beam currents as a function of $\mathrm{J}_{\mathrm{NK}}$, the time-mean neutralizer keeper current, for a $d_{N}$ of $0.193 \mathrm{~cm}$ and a $\dot{\mathrm{m}}_{\mathrm{N}}$ of 6.5 milliamps. The curve for the beam fluctuations is such that for all values of $\bar{J}_{\mathrm{NK}}$ the ratio $\sqrt{\overline{\mathrm{j}_{\mathrm{B}}}} \mid \overline{\mathrm{J}}_{\mathrm{B}}$ is greater than the ratio $\sqrt{\overline{\mathrm{j}_{\mathrm{NK}}^{2}}} \mid \bar{J}_{\mathrm{NK}} \cdot$ As $\bar{J}_{\mathrm{NK}}$ decreases to $0.2 \mathrm{amp}$ the ratio $\sqrt{\overline{j_{B}^{2}}} / \bar{J}_{B}$ increases steply to 0.160 . This increase in the level of beam current fluctuations may simply be the result of an insufficient number of electrons to effectively neutralize the beam at low values of $\mathrm{J}_{\mathrm{NK}}$.

To this point there has been no mention of the rms magnitudes of the fluctuations in discharge current and voltage. Because the steady-state conditions in the discharge were controlled to be constant at $J_{E}$ of $0.70 \mathrm{amp}$ and $\Delta V_{I}$ of 38.0 volts, it was expected that the rms magnitudes of the discharge current and voltage would not change significantly over the serles of tests. In fact, there was some variation and over the series of tests, $\sqrt{\overline{\Delta v_{I}^{2}}}$ the rms magnitude of the discharge voltage was $1.16 \pm 0.17$ volts or $\sqrt{\Delta v_{I}^{2}} / \frac{}{\Delta V_{I}}$ was $0.03 \pm 0.0044$. Over the series of tests $\sqrt{\mathrm{j}_{\mathrm{E}}^{2}}$, the rms magnitude of the discharge current, was $0.0447 \pm 0.0079$ amp or $\sqrt{\overline{j_{E}^{2}}} / \bar{J}_{E}$ was $0.0064 \pm 0.0011$. Examination of these data in some detail did not reveal any particular trend with any of the neutralizer parameters being 
varied or the consequent variation in the neutralizerbeam coupling. Although the variation in the rms magnitudes of the discharge voltage and current was small, its source or reasons for being are unexplained.

Knowledge of $\mathrm{V}_{\mathrm{g}}$ fluctuations may be significant to determine the import, if any, of thruster operation on the spacecraft interface in relation to system operations, spacecraft charging, etc. The quantity $\mathrm{V}_{\mathrm{g}}(\mathrm{t})$ is the potential between the neutralizer common and facility ground, and is indicative of the degree of coupling between the neutralizer and the beam plasma. In space, this coupling potential represents the potential between the spacecraft and the space plasma surrounding it. Perturbations or oscillations on this coupling potential, If they were to exist, could be significant for mission operation.

A limited investigation of the fluctuations or timevarying property of the neutralizer floating potential $\left(-v_{g}\right)$ was done near the end of the series of tests. These initial measurements of the $\sqrt{\overline{v_{g}^{2}}}$, the rms magnitude of the neutralizer floating potentlal, were made for neutralizer keeper hole diameters, $d_{N}$, of 0.193 and $0.257 \mathrm{~cm}$. The neutralizer keeper current, $\mathrm{J}_{\mathrm{NK}}$, and flow rate, $\dot{\mathrm{m}}_{\mathrm{N}}$, were $0.45 \mathrm{amp}$ and 7.0 milliamperes, respectively. For the $d_{N}$ of $0.193 \mathrm{~cm}$, the value of $\sqrt{\overline{v_{g}^{2}}}$ was 0.275 and the value of $\sqrt{\overline{v^{2}}} /\left|\bar{v}_{g}\right|$ was 0.031 . For the $\mathrm{d}_{\mathrm{N}}$ of $0.257 \mathrm{~cm}$, the value of $\sqrt{\overline{\mathrm{v}^{2}}}$ was 0.179 and the value of $\sqrt{\overline{\mathrm{v}_{\mathrm{g}}^{2}}} /\left|\overline{\mathrm{V}}_{\mathrm{g}}\right|$ was 0.021 . These results for the $\mathrm{v}_{\mathrm{g}}$ fluctuations are similar in their values to those for the neutralizer keeper and beam current fluctuations. In addition, these values of $\sqrt{\overline{\mathrm{v}_{\mathrm{g}}^{2}}} /\left|\overline{\mathrm{v}}_{\mathrm{g}}\right|$ appear to be sufficiently small as to not appreciably effect the behavior of the spacecraft potentials.

\section{Spectral Data}

The spectra of the fluctuations in terms of the relative amplitude in decibels as a function of frequency are given in Fig. 8 for a typical operating condition of the thruster with a $J_{\mathrm{NK}}$ of $0.45 \mathrm{amp}, \dot{\mathrm{m}}_{\mathrm{N}}$ of 7.0 milliamps and $\mathrm{d}_{\mathrm{N}}$ of $0.157 \mathrm{~cm}$. Figure $8(\mathrm{a})$ presents this type of spectrum for the fluctuations of the discharge current and for the fluctuations of the discharge voltage; Fig. 8 (b) for the fluctuations of the neutralizer keeper and ion beam currents. The spectra of the discharge voltage fluctuations in Fig. 8(a) is mainly characterized by a highest peak at about $78 \mathrm{kHz}$ and on both sides of this peak there is a fall-off in amplitude. The fall-off in amplitude as the frequencies decrease below the peak frequency is comparable to that found in Ref. 2 for the discharge fluctuations. If the portion of the spectrum between $10^{5}$ and $10^{6} \mathrm{~Hz}$ is examined, the dependence on frequency is $f^{-2.1}$. Thus, on the basis of the discharge current fluctuation spectrum alone there is some resemblance here to the spectrum observed in plasma turbulence experiments which exhibit a $\mathrm{f}^{-2.5}$ dependence. ${ }^{(3-5)}$

In Fig. 8(a) the spectrum for the fluctuations in discharge voltage exhibits the same maximum or peak of grcatest amplitude at $78 \mathrm{kHz}$ as the spectrum for the discharge current fluctuations. However, elsewhere in frequency the two spectra are dissimilar. In the case of the discharge voltage, for frequencies less than the peak frequency, the fall-off is small as the frequency decreases, only $12 \mathrm{~dB}$ from the peak frequency to $200 \mathrm{~Hz}$. Above the peak frequencies there is a fall-off in amplim tude with increasing frequency of an $\mathrm{f}^{-4.5}$ dependence. There is a smaller peak $(-17.5 \mathrm{~dB})$ at about $1.6 \mathrm{MHz}$ which may result from a beam/power supply interaction as the particular peak is also evident in the spectra for the beam current and neutralizer current fluctuations.

The frequency at which the peak occurs in Fig. 8(a) is so dominant that its physical origin should be considered. Quick calculation reveals that of the plasma resonances or oscillations observed in similar plasmas, there are at least three possibilities. ${ }^{(6-8)}$ These possibilities are ion-acoustic resonances, resonances due to $\overrightarrow{\mathbf{E}} \times \overrightarrow{\mathbf{B}}$ particle drifts, and oscillations due to effects of anomalous diffusion. Without a more complete knowledge of the plasma it will not be possible to compare quantitatively the observed peak with ealculated values. However, by making some appropriate assumptions on the values expected for the plasma parameters, at least a qualitative comparison can be presented.

The ion-acoustic resonance can be calculated by using $v_{\text {th }}=\sqrt{\mathrm{kT}_{\mathrm{e}} / \mathrm{M}_{\mathrm{i}}}$ for the characteristic velocity where $T_{e}$ is the electron temperature and $M_{i}$ is the mass of the mercury ion. A characteristic frequency can be obtained by setting, $v_{t h} / \ell$ where $\ell$ is a characteristic length in the discharge. If a $\mathrm{T}_{e}$ of $10 \mathrm{eV}$ and an $\ell$ of $3 \mathrm{~cm}$ is assumed, then the characteristic frequency obtained is $73 \mathrm{kHz}$ which is quite close to the observed $78 \mathrm{kHz}$. However, this was intentionally done by choice of the $\mathrm{T}_{\mathrm{e}}$ and $\ell$ values. The only questions that remaln to be resolved are obviously the correctness of the assumptions. Certainiy, they are reasonable ones.

The $\overrightarrow{\mathrm{E}} \times \overrightarrow{\mathrm{B}}$ particle drift resonance can be calculated by using $v_{d}=10^{8} \mathrm{E} / \mathrm{B}$ for the characteristic velocity where $\mathrm{E}$ is in volts $/ \mathrm{cm}$ and is assumed to be perpendicular to $\mathrm{B}$ given in gauss. This characteristic frequency can be also obtained by setting $v_{d} / \ell$ where $\ell$ is a characteristic length in the discharge. If it is assumed that $\mathrm{E}$ has a value of 0.1 volts $/ \mathrm{cm}, \mathrm{B}$ has a value of 30 gauss and $\ell$ is $3 \mathrm{~cm}$, then characteristic frequency is obtained as $110 \mathrm{kHz}$. It is difficult to justify lowering the value of $\mathrm{E}$ or increasing the value of $l$ to 
obtain better agreement with the observed frequency. Consequently, the possibility of the $\overrightarrow{\mathrm{E}} \times \overrightarrow{\mathrm{B}}$ particle drift resonance being the cause of the observed peak in Fig. 8 (a) is remote.

A calculation of the plasma resonance resulting from anomalous diffusion ${ }^{(8)}$ will not be attempted because of the complete lack of the needed plasma parameters such as the ion temperature and the critical magnetic field at which there is an onset of the anomalous diffusion. There may be factors other than those mentioned above which affect the resonance in the plasma such as geometry and interaction of the thruster plasma with the power processor, but these are not considered here.

In Fig. 8 (b) which gives the spectrum of the neutralizer keeper current fluctuations the maximum amplitude occurs at about $1.65 \mathrm{MHz}$. There are lesser peaks, not as sharp, at 175 and $800 \mathrm{kHz}$. Further discussion of the neutralizer spectrum will be deferred until additional data on the neutralizer fluctuations has been presented.

Figure 8(b) also gives the spectrum of the beam current fluctuations which has a broad maximum at the very low frequencies, about 0.8 to $1.0 \mathrm{kHz}$. Then, as the frequencies increase above this point, there is a gradual fall-off in amplitude with several smaller peaks being indicated. There is a peak with an amplitude of $-28 \mathrm{~dB}$ at a frequency of $780 \mathrm{kHz}$. Another peak that can be identified is the one with an amplitude of $-26.5 \mathrm{~dB}$ at a frequency of $1.65 \mathrm{MHz}$ which corresponds to the maximum of the spectrum of the neutralizer keeper current fluctuations. This is the result of neutralizer-beam interaction. The essential point about the beam current fluctuation spectrum is that it is not dominated by the resonance of the discharge chamber fluctuations (frequency of $78 \mathrm{kHz}$ ) as was the case for the results of the study on the $30-\mathrm{cm}$ thruster. ${ }^{(2)}$

The spectral distribution of neutralizer keeper current fluctuations is shown in Fig. 9 for three values of neutralizer floating potential. The spectra are given as relative amplitude in decibels as a function of frequency in Hertz. Curve $A$ is the spectrum for a "good coupling" of the beam and neutralizer currents with $\mathrm{V}_{\mathrm{g}}$ of -8.5 volts. Curve B shows an "intermediate coupling" at a $\mathrm{V}_{\mathrm{g}}$ of -18.5 volts and curve $\mathrm{C}$ for a "poor coupling" mode with $\mathrm{V}_{\mathrm{g}}=-39$ volts. As the coupling of the beam and neutralizer progresses from "good" to "poor" the spectrum of the neutralizer keeper current fluctuations changes. The relative magnitude of the peaks at the various frequencles are different. For curve $A$ there are smaller and broad peaks at frequencies of $-9.3 \mathrm{~dB}$ at $200 \mathrm{kHz}$ and $-5 \mathrm{~dB}$ at $620 \mathrm{kHz}$ and a maximum at 1.85 MHz. The "poor coupling" mode shows that the maximum response occurs at $118 \mathrm{kHz}$. There also are magnitude peaks at the first and second harmonics of the frequency of the maximum peak. These two frequencies are 238 and $375 \mathrm{kHz}$, respectively. The spectrum for the "Intermediate coupling" mode shows a set of peaks at the lower and higher frequencles simllar to those discussed above. These peaks are approximately of the same magnitude.

It is evident from the results of Fig. 9 that the characteristic shapes for the spectra of neutralizer kecper current fluctuations are a function of how well the neutralizer is coupling to the beam. The frequencies at which the peaks occur in the three curves of Fig. 9 are not expected to be the same because the neutralizer parameters are not the same. For example it is seen that the lowest frequency in curve $B$ is $75 \mathrm{kHz}$ and in curve $\mathrm{C}$ it is $118 \mathrm{kHz}$. However, the peak occurring at a frequency of about $1.65 \mathrm{MHz}$ is the same for the three curves.

The lower frequencies of curves $B$ and $C$ in Fig. 9 are characterized as having a fundamental frequency plus at least a first harmonic. The pressures in the cathode, at the cathode orffice and just downstream of the orifice are sufficiently large to permit the plasma to be considered to be in the continuum regime. This permits the inclusion of another resonance to be considered and this is a nonplasma or acoustic resonance involving only the neutrals. With the assumption that the neutrals possess a temperature equal to the cathode tip temperature of $700^{\circ} \mathrm{C}$, the characteristic or acoustic velocity is $2.59 \times 10^{4} \mathrm{~cm} / \mathrm{sec}$. If a characteristic dimension is taken as $2 \ell$ where $l$ is the cathode-to-keeper axial gap and is $0.109 \mathrm{~cm}$ for the data of Fig. 9, then a typical acoustic resonance frequency is $112 \mathrm{kHz}$. This is a value for the frequency that is at or very close for a considerable number of the spectra measured, In addition, for one test the cathode-to-keeper gap was changed to $0.175 \mathrm{~cm}$. This ratio of the two gaps is $\ell_{1} / \ell_{2}=$ $0.109 / 0.175=0.624$. The inverse ratio of the fundamental lower frequency for the two gaps was measured as $f_{2} / f_{1}=115 / 168=0.685$. Thus, this additional test is a further indication of the possibility that the acoustic resonance is responsible for the peaks at the lower frequencies of spectrum of fluctuations of the neutralizer keeper current. What is inexplicable at this point is the suppression of this mode at "grood coupling" conditions if in fact the responstble resonance is the acoustic resonance.

Another possibility responsible for the peaks in the spectra of Fig. 9 is the ion-acoustic resonance considered earlier in the case of the discharge chamber fluctuations. The range of electron temperatures considered for the neutralizer are obtalned from Ref. 9. Possible values of electron temperature to be considered here are 0.86 to $2.5 \mathrm{eV}$. If a $\mathrm{T}_{\mathrm{e}}$ of $0.86 \mathrm{eV}$ is con- 
sidered first for a characteristic length of $0.109 \mathrm{~cm}$ (the cathode-to-keeper gap) an ion-acoustic frequency of $680 \mathrm{kHz}$ is obtalned. Using a characteristic length of $1.0 \mathrm{~cm}$ (diameter of region between cathode and keeper) yields an ion-acoustic frequency of $75 \mathrm{kH}$. Increasing the $T_{E}$ to $2.5 \mathrm{eV}$ yields frequencies of $1.01 \mathrm{Mfz}$ and $270 \mathrm{kHz}$, respectively, for characteristic lengths of 0.109 and $1.00 \mathrm{~cm}$. Thus, it is seen that the ionacoustic resonance could possibly account for most of the peaks in the spectra. For peaks occurring from 1.65 to $1.85 \mathrm{MHz}$ (curve C of Fig. 8(b) and Fig. 9), the characteristic length would have to be much smaller, such as the diameter of the cathode orifice of $0.25 \mathrm{~cm}$. Using this assumption and a $T_{E}$ of $0.86 \mathrm{eV}$ does yield a frequency of about 1.6 MHz. However, because the actual value of $T_{E}$ for this particular data is not known this matter will not be pursued any further.

The spectral distribution of the ncutralizer keeper current fluctuations for two values of time-mean neutralizer keeper current is shown in Fig. 10. Curve A in Fig. 10 is for a $J_{\mathrm{NK}}$ of $0.45 \mathrm{amp}$ and $\mathrm{V}_{\mathrm{g}}=-9$ volts at a $\mathrm{d}_{\mathrm{N}}$ of $0.157 \mathrm{~cm}$ and $\dot{\mathrm{m}}_{\mathrm{N}}=7.0$ milliamps. Curve $\mathrm{B}$ is the result of changing $\mathrm{J}_{\mathrm{NK}}$ to $0.3 \mathrm{amp}$ and holding the other neutralizer parameters constant. The resulting change in $\mathrm{V}_{\mathrm{g}}$ is to -26 volts. This data in Fig. 10 clearly illustrates with only one neutralizer parameter changing how the shape of the spectra changes significantly in going from a "good coupling" mode to a less desired mode of neutralizer and beam coupling as depicted by curves $A$ and $B$, respectively, in Fig. 10 .

The data for the $\mathrm{V}_{\mathrm{g}}$ fluctuations were obtained near the end of the series of tests. Because of the fundamental character of the floating potential fluctuations with respect to spacecraft interface, the preliminary results are given in Fig. 11. This figure allows a comparison of the spectrum of the neutralizer floating potential fluctuations with those for the neutralizer keeper and beam current fluctuations. Curves A, B, and C in Fig. 11 give the spectra for the fluctuations of neutralizer keeper current, beam current and neutrallzer floating potential, respectively. The neutralizer keeper hole diameter, $\mathrm{d}_{\mathrm{N}}$, of $0,258 \mathrm{~cm}$ was the largest tested and represents a "good coupling" situation with a $\mathrm{V}_{\mathrm{g}}$ of -8.5 volts, $\mathrm{J}_{\mathrm{NK}}$ of 0.45 ampere and $\dot{\mathrm{m}}_{\mathrm{N}}$ of 7.0 milliamps.

The spectrum given by curve $A$ is representative of that previously presented for a "good coupling" situation with a maximum amplitude peak occurring at $1.1 \mathrm{MHz}$. The lesser peaks in the spectrum occur at $140 \mathrm{kHz}$, 1.9 and $3,3 \mathrm{MHz}$. The spectrum given by curve $B$ for the beam current fluctuations is essentially similar to that given in Fig. $8(\mathrm{~b})$. The spectrum given by curve $B$ has a broad maximum from the lowest measured frequency $(200 \mathrm{~Hz})$ up to about $5.5 \mathrm{kHz}$ with a fall-off in amplitude as the frequency increases. There is a lesser peak at a frequency of $80 \mathrm{kHz}$, corresponding to the discharge chamber resonance. There are still lesser peaks at frequencies of 1.1 and $1.9 \mathrm{MHz}$. These lesser peaks correspond with frequencies of peaks in the spectrum of the fluctuations of the neutralizer keeper current given by curve $A$. The spectrum given by curve $C$ for the neutralizer floating potential fluctuations exhibits peaks seen in both the spectra of neutralizer keeper current and beam current fluctuations. A broad maximum in curve $\mathrm{C}\left(\mathrm{V}_{\mathrm{g}}\right.$ fluctuations) is from the lowest frequency measured $(200 \mathrm{~Hz})$ to about $1 \mathrm{kHz}$. Again there is a falloff in amplitude with increasing frequency to about 100 $\mathrm{kHz}$. There are several peaks in this frequency range one of which occurs at the discharge chamber resonance. Between $100 \mathrm{kHz}$ and $1 \mathrm{MHz}$, the spectrum is nearly flat with a relative amplitude of about $-32 \mathrm{~dB}$. Then, as the frequency increases, the level of the spectrum increases such that at peaks which occur at $1.3,2.0$, and $2.8 \mathrm{MHz}$ the relative amplitudes are $-11,-5$, and $-11.5 \mathrm{~dB}$, respectively.

\section{Correlations of the Fluctuations}

To determine the geometric size of the beam that is fluctuating as a whole ("in-phase"), cross-correlations or space-time correlations were measured (see appendix B of Ref. 2). As indicated in the Ref. 2, the correlation curves were obtained in terms of $R$, the correlation coefficient, as a function of $\tau$, the time delay between the fluctuations measured at two points in the beam. The cross-correlation curves had a maximum, $\mathbf{R}_{\text {max }}$, usually near $\tau=0$ which implied that (for the given probe separation distances and time-resolvability of the correlation analyzer) the fluctuations did not have measurable convective velocities, ${ }^{(10)}$

Figure 12 gives the variation of $R_{\max }$ of the correlations of the beam fluctuations as a function of radial probe separation distance. Also included in the figure are data given in Fig. 18 of Ref. 2 which were taken in the beam of a $30-\mathrm{cm}$ thruster at a distance from the accelerator grid of $17.8 \mathrm{~cm}$. In the case of both the data from Ref. 2 and that presented herein, one probe remained at $r=0$ and the other probe varied across the radius. To permit a comparison of the two sets of data, the radial separation, $\zeta_{r}$, is divided by $r_{T}$, the thruster radius. From Fig. 12 it is seen that the values of $R_{\max }$ are quite large (i. e., $\geq 0.50$ ) throughout the radial extent of the beam. The variation of $R_{\max }$ with ${ }^{5} \mathrm{r}^{/ \mathrm{r}_{\mathrm{T}}}$ is about the same for both the 8-cm and the 30$\mathrm{cm}$ thrusters. This signifies that the radial scale of the fluctuations is as large as the beam diameter. For the 8-cm it was not possible because of facility limitations to explore the behavior of $R_{\max }$ with axial separation of the two probes. Because of the similarity of the results for the radial probe scparation with Ref. 2 , there 
probably exists a like similarity of results for the axial probe separation.

As in Ref. 2, the relationship between the discharge current fluctuations and the beam fluctuations was studied by measuring the cross-correlations between these two fluctuations. Also measured was the crosscorrelations of the neutralizer keeper current fluctuations with the beam fluctuations. In addition the correlation of the fluctuations of the discharge voltage with the discharge current fluctuations was obtained. The results of these correlation measurements are presented in Table I which gives the ranges of values of the maximum correlation coefficients, $R_{\max }$ that were obtained. The first column in Table $I$ gives the range of values of $R_{\max }$ for the correlations of $\Delta v_{I}$ fluctuations and $f_{E}$ fluctuations. The $R_{\max }$ values vary from 0.72 to 0.78 which is essentially no variation over the series of tests since the time-mean discharge chamber parameters were held constant. The rather high value of $R_{\max }$ indicates that the fluctuations in current and voltage are a consequence of the same physical phenomena. However, it should be understood that this particular crosscorrelation is not only sensitive to the nature of the discharge chamber fluctuations themselves, but could also be sensitive to the nature of impedance of the power supplies as viewed from the thruster. Thus, altering power supply characteristics or their output impedance could produce diflerent results from those in the table.

The second column in Table I gives the range of values of $R_{\max }$ for the correlations of the neutralizer keeper current fluctuations, $j_{\mathrm{NK}}(t)$ with the beam fluo tuations, $j_{B}(t)$. The $R_{\max }$ values vary from 0.09 to 0.18 which is essentially similar to the range of values obtained in Ref. 2 for the 30-cm thruster. This result is not unexpected. In both Ref. 2 and here, the spectra of the beam and neutralizer keeper fluctuations are not similar (i.e., refer to Fig. 8(b) or Figs. 11(a) and (b) of this paper). The lack of similarity of the spectra always results in small eross-correlation coefficients by definition of the cross-correlation of two fluctuations.

The third column in Table I gives the range of values of $R_{\max }$ for the correlations of the discharge current fluctuations, $j_{E^{\prime}}$ (t) with the fluctuations of the beam current, $j_{B}(t)$. The $R_{\max }$ values vary from 0.08 to 0.23 which is much lower than the results obtained for the 30 -cm thruster presented in Ref. 2. There the $R_{\max }$ for the correlations of $j_{E}(t)$ and $j_{B}(t)$ varied from 0.7 to 0.9. Agaln referring to the spectra of the fluctuations yields the explanation for this difference in the values of $\mathbf{R}_{\text {max }}$ In Ref. 2, the spectra for the discharge current and beam fluctuations were nearly similar and hence the large values of $\mathbf{R}_{\max }$ are entirely reasonable for the results of Ref. 2, In the present work, spectra of the discharge current fluctuations and beam current fluctuations are not similar as can be seen by referring to
Figs, 8(a) and (b). The maximum amplitudes of the spectra occur at 78 and $0.9 \mathrm{kHz}$, respectively, for the discharge current and beam current fluctuations.

\section{Concluding Remarks}

An experimental investigation of the fluctuations or time-varying properties of an 8-cm electronbombardment thruster with a laboratory power supply console has been performed. The steady-state parameters of the thruster that were kept constant were beam current, $\mathrm{J}_{\mathrm{B}}$, of 72 milliamps, discharge voltage, $\Delta \mathrm{V}_{\mathrm{I}}$, of 38.0 volts, and discharge current, $J_{E}$, of $0.70 \mathrm{amp}$. Included in the study was a variation of the neutralizer parameters to observe the effect on the time-varying aspects of the coupling of the neutralizer with the beam. The results include the following:

1. Varying the neutralizer parameters including keeper hole diameter resulted in a wide variation of the ratio of the rms magnitude of the fluctuations to the time-mean of the beam current from 0.02 to 0.67 .

2. For situations of good or optimum coupling of the neutralizer to the beam, $-v_{g}$ being 12 volts or less, the ratio of the rms magnitude of the fluctuations to the time-mean of the beam current was 0.07 or less.

3. A relationship was found between the steady-state (time-mean) and the time-varying coupling of the neutralizer with the beam.

4. The ratio of the rms magnitude of the fluctuations to the time-mean neutralizer keeper current was found to depend strongly on the neutralizer parameters of the neutralizer keeper current, $J_{N K}$, the flow rate, $\dot{\mathrm{m}}_{\mathrm{N}}$, and the keeper hole diameter, $\mathrm{d}_{\mathrm{N}}$.

5. Maxima of the spectra for the fluctuations in discharge current and voltage occurred at about $78 \mathrm{kHz}$ and could possibly be the result of the presence of ionacoustic waves in the discharge plasma or other plasma resonances.

6. The maxima of the spectra of the beam current fluctuations did not depend on the discharge plasma fluctuations. This fact along with the cross-correlation results indicates that the discharge current fluctuations did not directly contribute to the beam current fluctuations.

7. The cross-correlations of the beam fluctuations indicate that the radial scale of the fluctuations is as large as the beam diameter.

8. The rms magnitudes, spectral and crosscorrelation results of the neutralizer keeper current fluctuations indicate that the neutralizer contributions to the beam fluctuations could be small (for good neutrallzer-to-beam coupling) but not negligible and appear mostly in the higher frequency range measured. 


\section{Appendix - Symbols}

\section{$\mathrm{d}_{\mathrm{N}}$}

f

$I(t)=\overline{\mathrm{I}(\mathrm{t})}+\mathrm{i}(\mathrm{t})$

$i(\mathbf{t})$

$J_{B}(t)=\overline{J_{B}(t)}+j_{B}(t)$

$\mathbf{j}_{\mathrm{B}}(\mathbf{t})$

$J_{E}(t)=\overline{J_{E}(t)}+j_{E}(t)$

$\mathbf{j}_{\mathrm{E}^{(t)}}$

$J_{N K}(t)=\overline{J_{N K}(t)}+j_{N K}(t)$

$\dot{j}_{N K}(t)$
$\dot{m}_{N}$
$R_{\max }$
$r$
$r_{T}$
$v_{g}(t)=\overline{v_{g}(t)}+\Delta v_{g}(t)$
$v_{g}(t)$
$\Delta v_{I}(t)=\overline{\Delta v_{I}(t)}+\Delta v_{I}(t)$
$\Delta$
$s_{r}$

neutralizer kecper holc diameter, cm

frequency, $\mathrm{Hz}$

sum of time-mean and timevarying beam probe currents, milliamps

time-varying probe current, milliamps

sum of time-mean and timevarying beam currents, milliamps

time-varying beam current, milliamps

sum of the time-mean and timevarying discharge (emission) currents, amps

time-varying discharge (emission) current, amps

sum of time-mean and timevarying neutralizer keeper currents, amps

time-varying neutralizer keeper curxent, amps

equivalent neutralizer flow rate, milliamps

maximum correlation coefficient at a given probe separation

radial probe distance from grid center, cm

radius of thruster, $\mathrm{cm}$

sum of time-mean and timevarying neutralizer floating potential, volts

time-varying neutralizer floating potential, volts

sum of time-mean and timevarying discharge voltages, volts

time-varying discharge voltage, volts

axial probe distance from gridcenter, cm

radial separation distance between two probes, cm
References

1. "g-Centimeter Mercury Ion Thruster Systems Technology, "ALAA Paper 74-1116, San Diego, Calif., 1974.

2. Serafint, J. S. and Terdan, F. F., "Plasma Fluc tuations in a Kauf man Thruster, "Journal of Spacecraft and Rockets, Vol. 11, No. 11, Nov. 1974, pp. $752-758$ (also AIAA paper 73-1056).

3. Serafini, J, S., "Some Observations on the Relationship between Instabilities and Turbulence in Plasma," presented at American Physical Society, Annual Meeting, 10th, Miami, Fla., Nov. 13-16, 1968 (also TM X-52501, 1968, NASA).

4. Chen, F. F., "Spectrum of Low- $\beta$ Plasma Turbulence, "Physical Review Letters, Vol. 15, No. 9, Aug. 1965, pp. 381-383.

5. Tchen, C. M., "A Caseade Theory of Turbulence," Bulletin of the American Physical Society, Vol. 13, No. 5, May 1968, p. 803.

6. Kobayashi, M, and Takagi, A., "Oscillations in a Duoplasma Ion Source, " Presented at Second Symposium on Ion Sources and Formation of Ion Beams, Berkeley, Calif., Oct. 22-25, 1974.

7. Poeschel, R. L., "A 2, $5 \mathrm{~kW}$ Advanced Technology Ion Thruster," Aug. 1974, Hughes Research Laboratories, Malibu, Calif.; also CR-134687, 1974, NASA.

8. Cohen, A. J., "Onset of Anomalous Diffusion in Electron-Bombardment Ion Thruster, "TN D-3731, 1966, NASA,

9. Fearn, D. G. and Philip, C. M., "An Investigation of Physical Processes in a Hollow Cathode Discharge, " ALAA Paper 72-416, Bethesda, Md., 1972 .

10. Serafini, J. S., "Wall-Pressure Fluctuations and Pressure-Velocity Correlations in a Turbulent Boundary Layer, " TR R-165, 1963, NASA.

'Table I. - Range of values of the maximum corrclation coefficients, $\mathbf{R}_{\max }$

\begin{tabular}{|c|c|c|}
\hline $\begin{array}{c}\text { For correlations } \\
\text { of } \Delta v_{1}(t) \text { and } j_{E^{(t)}}\end{array}$ & $\begin{array}{c}\text { For correlations } \\
\text { of } j_{N^{(t)}} \text { and } j_{B^{(t)}}\end{array}$ & $\begin{array}{c}\text { For correlations } \\
\text { of } j_{E^{(t)} \text { and } j_{\mathrm{B}}(t)}\end{array}$ \\
\hline 0.72 & 0.09 & 0.08 \\
to & to & to \\
0.78 & 0.18 & 0.23 \\
\hline
\end{tabular}




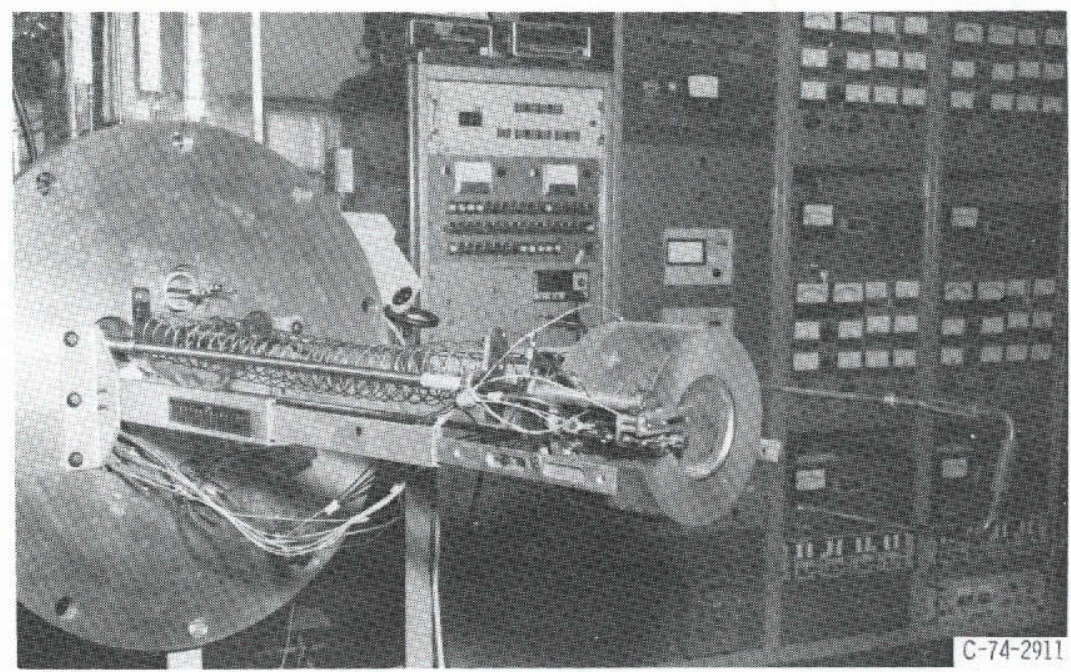

Figure 1. $-8-\mathrm{cm}$ Mercury ion thruster mounted on flange.

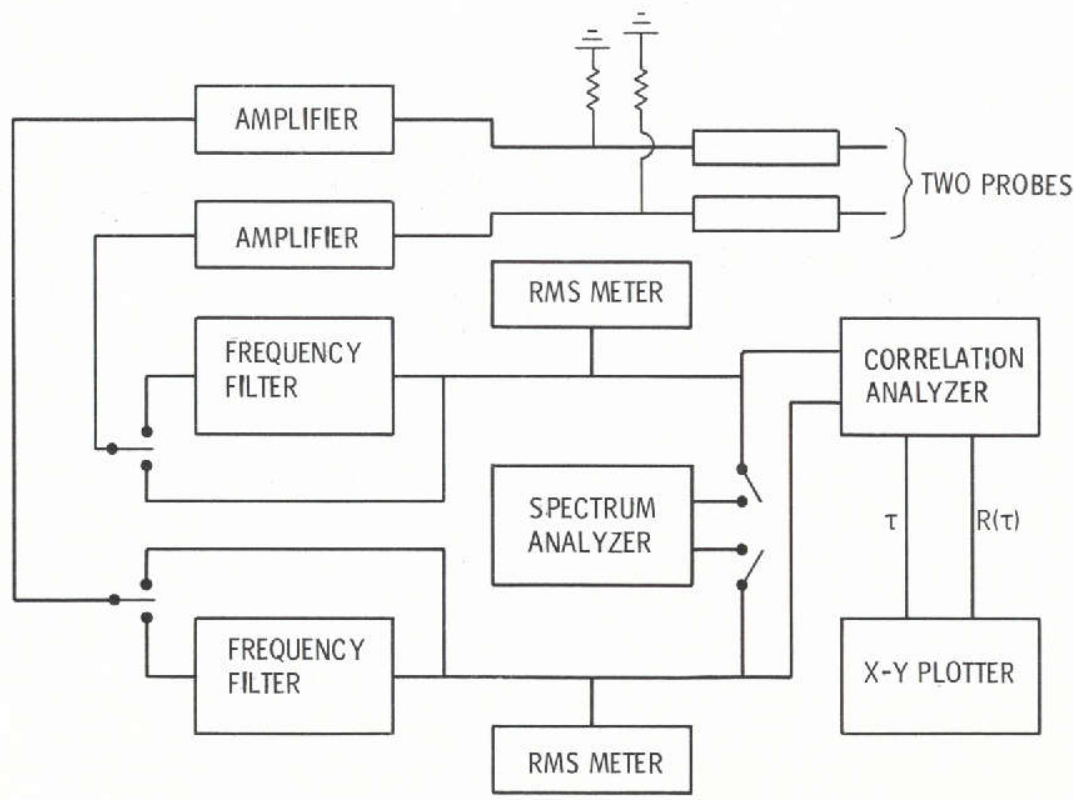

Figure 2. - Schematic diagram of instrumentation used to obtain cross-correlations and spectra for plasma fluctuations. 


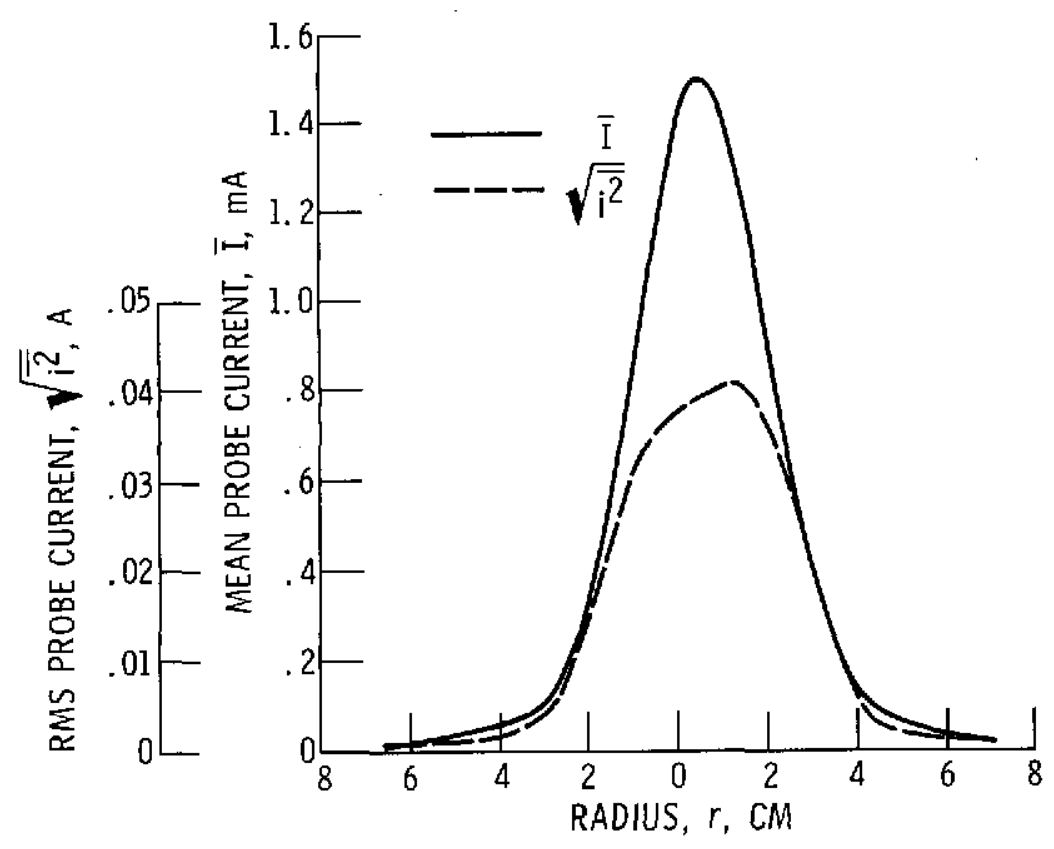

Figure 3. - A typical radial variation of the root-mean-square magnitude of the fluctuations and time-mean currents to the ion beam probe. $J_{B}=72 \mathrm{~mA} ; \Delta V_{I}=38 \mathrm{~V} ; J_{E}=0.70 \mathrm{~A}$.

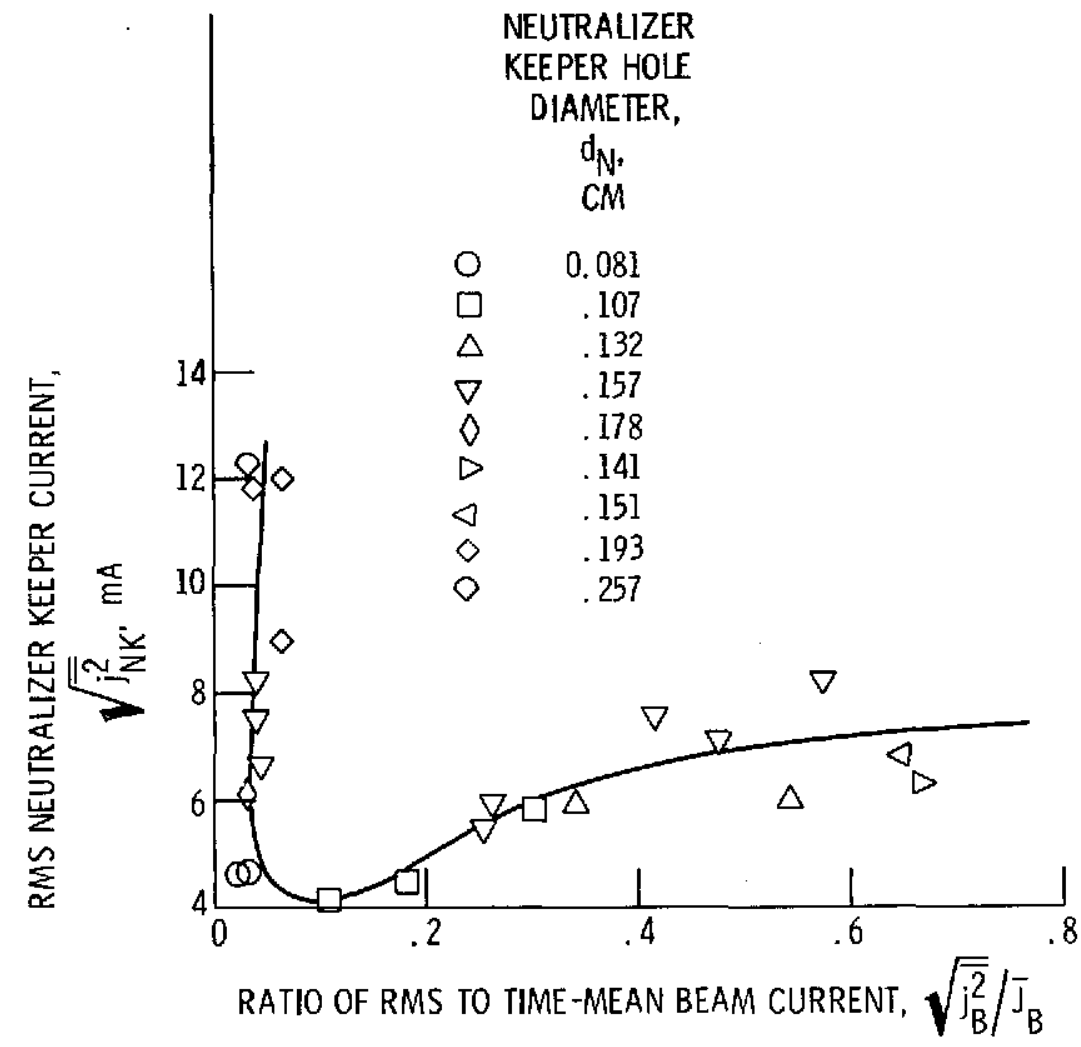

Figure 4. - Root-mean-square magnitude of fluctuations of neutralizer keeper current as a function of the ratio of the root-mean-square magnitude of fluctuations to timemean beam current for a range of neutralizer keeper hole sizes. 


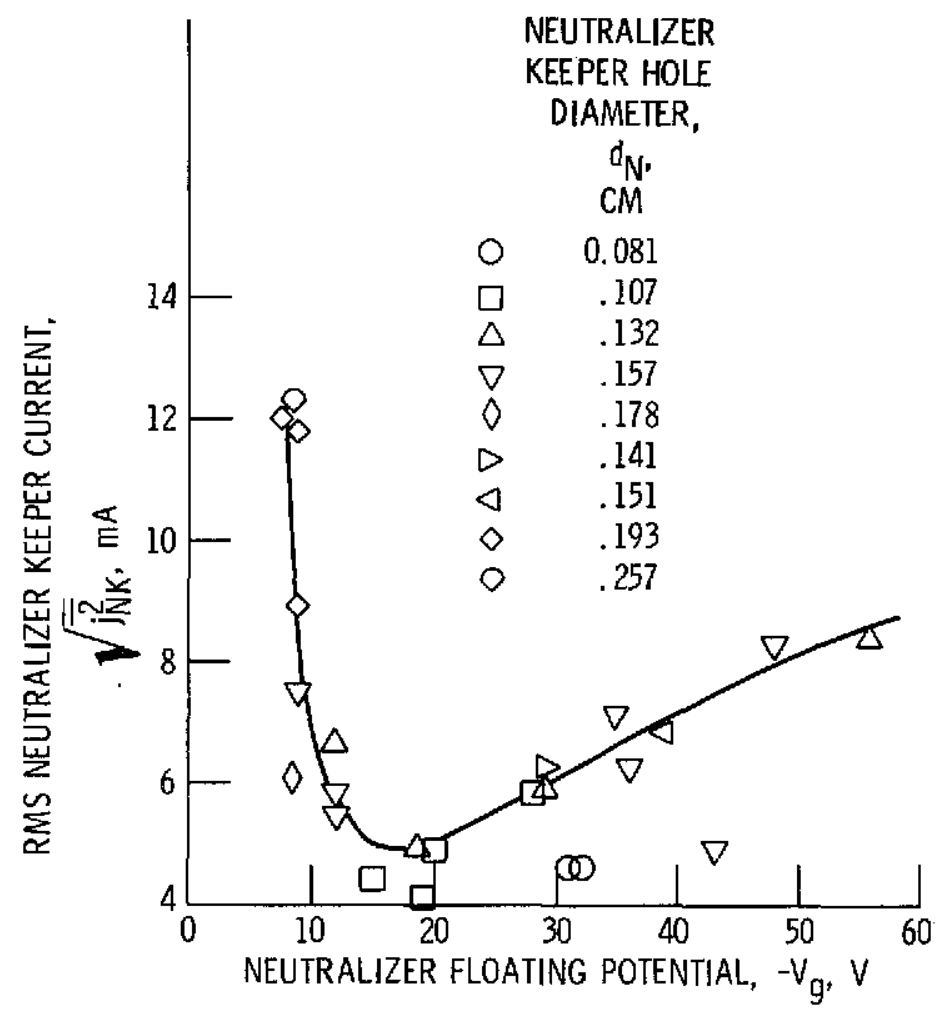

Figure 5. - Root-mean-square magnitude of fluctuations of neutralizer keeper current as a function of the neutralizer floating potential, $v_{g}$, for a range of neutralizer keeper hole sizes.

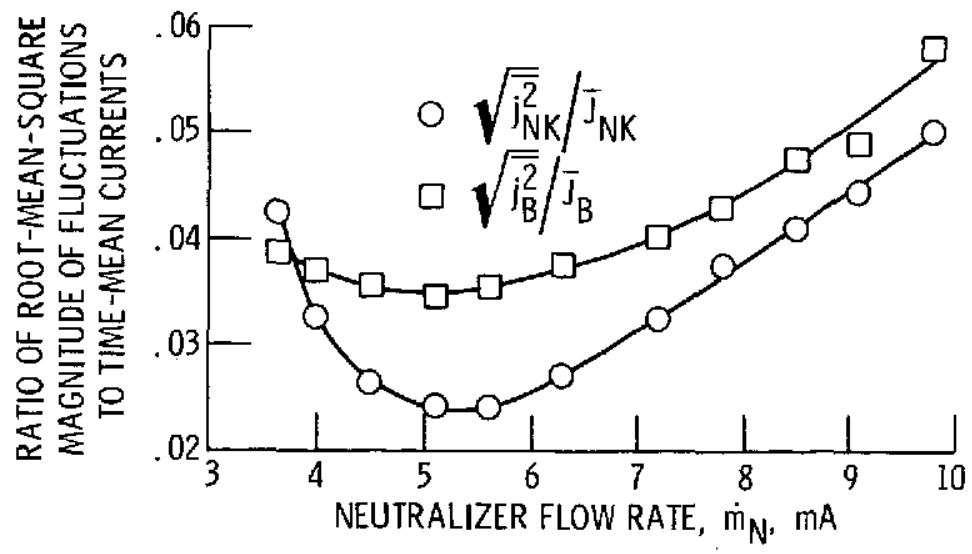

Figure 6. - Effect of varying the neutralizer flow rate on the ratio of root-mean-square magnitude of fluctuations to time-mean for the neutralizer keeper and ion beam currents. $J_{N K}=0.45 \mathrm{~A}$; diameter of neutralizer keeper hole, $d_{N}=0.193 \mathrm{~cm}$. 


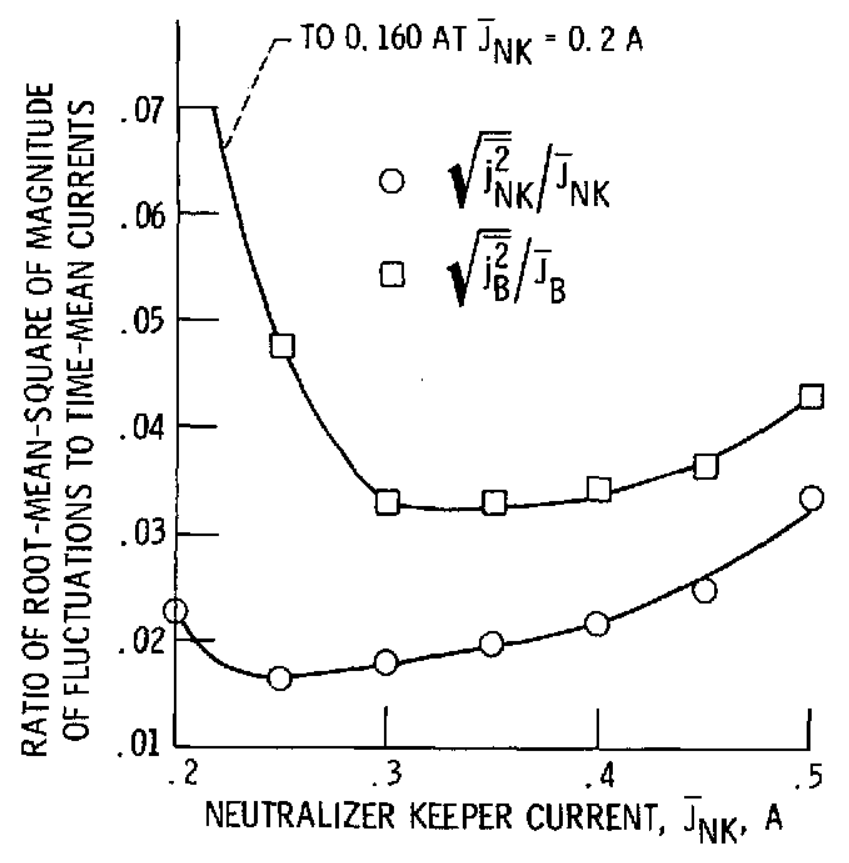

Figure 7. - Effect of varying the neutralizer keeper current on the ratio of root-meansquare magnitude of fluctuations to timemean for the neutralizer keeper and ion beam currents. Neutralizer flow rate, $\dot{m}_{N}=6.5 \mathrm{~mA}_{i}$ diameter of neutralizer keeper hole, $d_{N}=0.193 \mathrm{~cm}$.

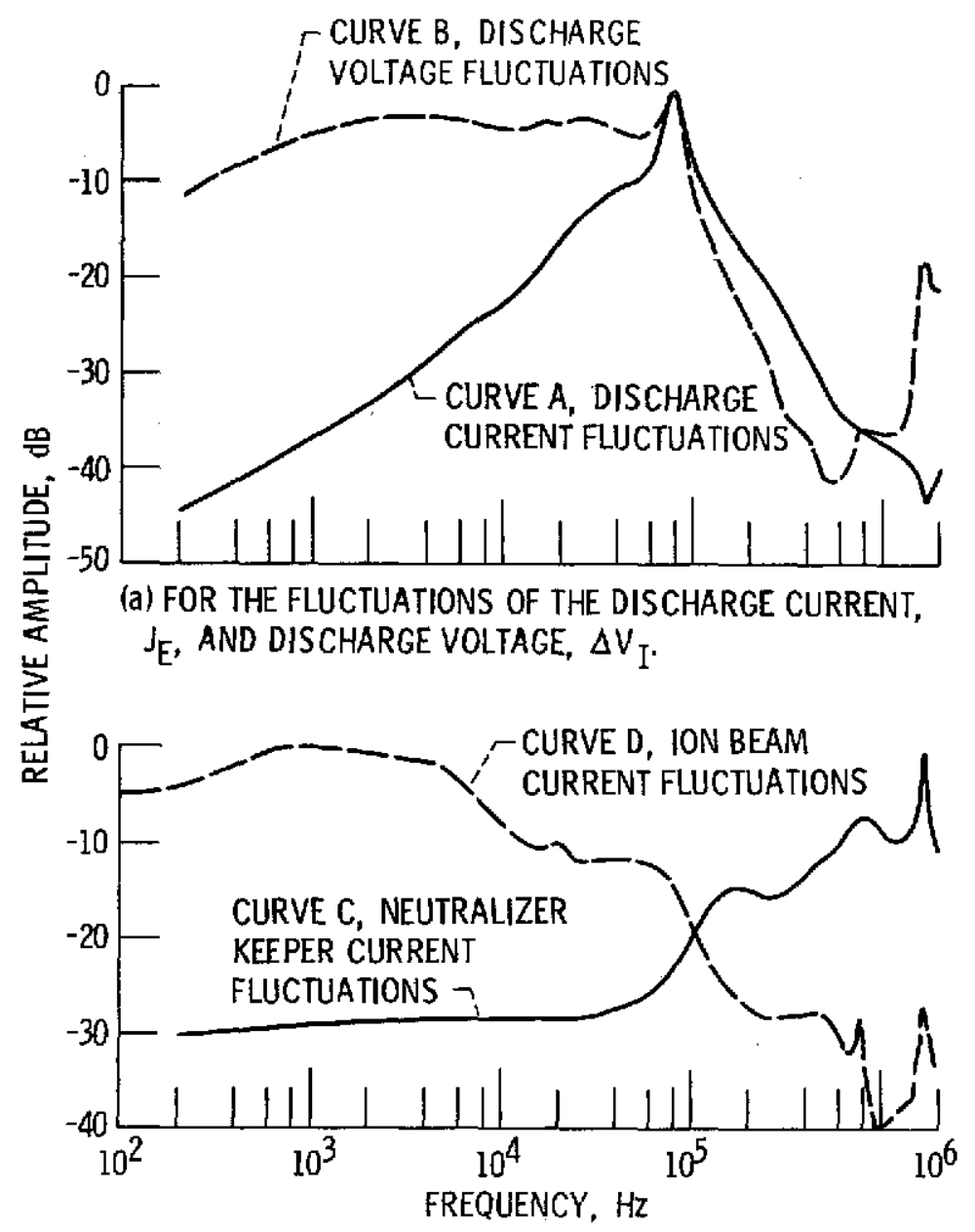

(a) FOR THE FLUCTUATIONS OF THE NEUTRALIZER KEEPER CURRENT, J JK, AND ION BEAM CURRENT, J $J_{B}$.

Figure 8. - Relative amplitude in decibels as a function of frequency of the fluctuations for a typical thruster operating condition. $J_{B}=72 \mathrm{~mA}_{;} \Delta \mathrm{V}_{\mathrm{I}}=38 \mathrm{~V} ; \mathrm{J}_{\mathrm{E}}=0.70 \mathrm{~A}$; neutralizer keeper hole diameter, $d_{N}=0.157 \mathrm{~cm}$. 


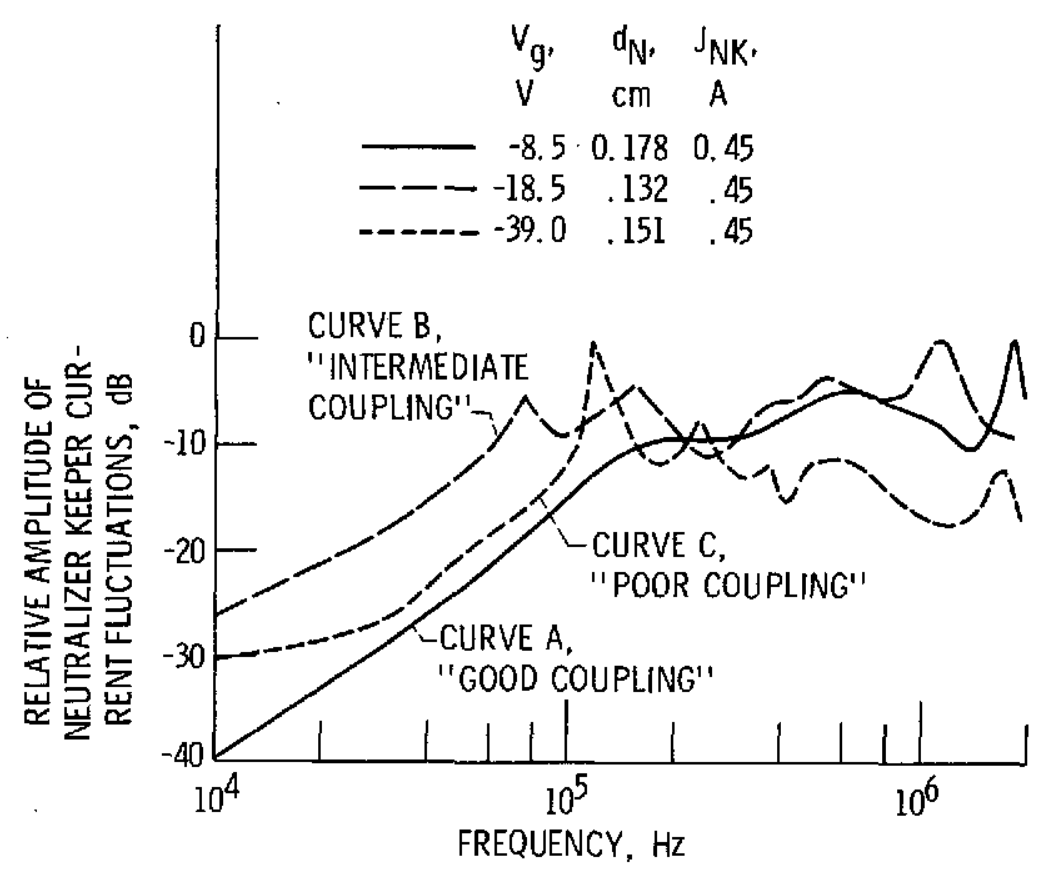

Figure 9. - Relationship between the neutralizer floating potential and the relative amplitude in decibels as a function of frequency for the neutralizer keeper current fluctuations.

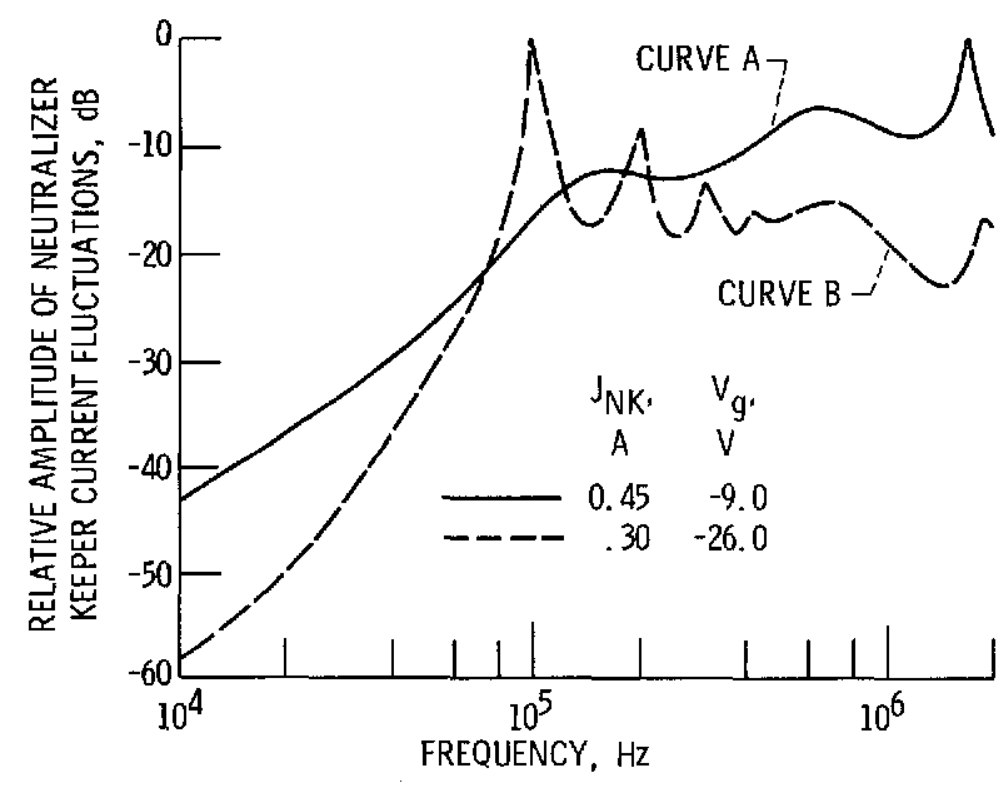

Figure 10. - Effect of varying the neutralizer keeper current on the relationship between the neutralizer floating potential and the relative amplitude in decibels as a function of frequency for the neutralizer keeper current fluctuations. Neutralizer keeper hole diameter, $\mathrm{d}_{\mathrm{N}}=0.157 \mathrm{~cm}$. 


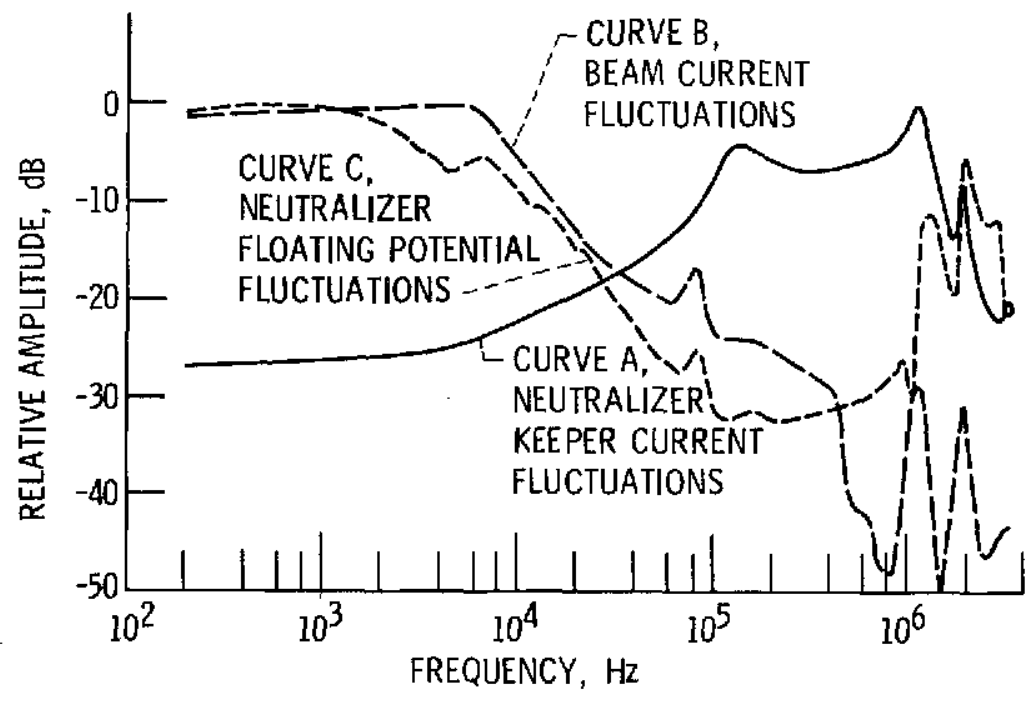

Figure 11. - Comparison of the relative amplitude as a function of frequency for the fluctuations of neutralizer keeper current, the ion beam current, and the neutralizer floating potential. Neutralizer keeper hole diameter, $d_{N}=0.258 \mathrm{~cm}$.

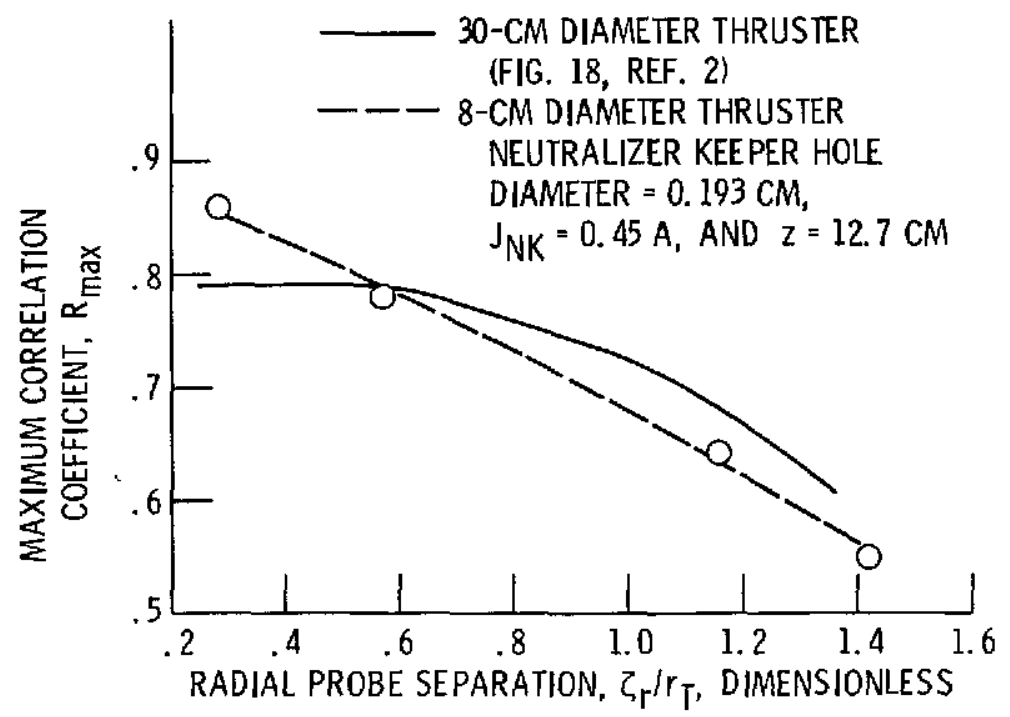

Figure 12. - Variation of $R_{\max }$ of the beam fluctuations as a function of the radial probe separation and comparison with results of Ref. 2 for the $30-\mathrm{cm}$ thruster. 